\title{
WATER IS THE MEANS, MEDIUM AND MESSAGE OF LIFE
}

\author{
MAE-WAN HO \\ Institute of Science in Society, UK.
}

\begin{abstract}
Everyone knows water is essential for life, and generations of scientists have marvelled at the properties of water that make it especially fit for life. Yet this simple, ubiquitous chemical compound has remained completely mysterious until quite recently. New evidence is beginning to unlock the mysteries. Liquid water is quantum coherent even at ordinary temperatures and pressure. It associates with macromolecules and membranes in a liquid crystalline configuration that enables enzymes and nucleic acids to function as quantum molecular machines that transform and transfer energy at close to $100 \%$ efficiency. Liquid crystalline water at interfaces also provides the excitation energy that enables it to split into hydrogen and oxygen in photosynthesis, simultaneously generating electricity for intercommunication and for the redox chemistry that ultimately powers the entire biosphere. Water is the means, medium and message of life; 'the rainbow within that mirrors the one in the sky'.

Keywords: Liquid crystalline water, proton conduction, quantum coherence, quantum jazz, resonant energy transfer.
\end{abstract}

\section{INTRODUCTION}

More than 20 years ago, I peered down a polarizing light microscope and saw a rainbow dancing inside a worm (Fig. 1). It took a while to decipher the meaning of that vision.

Typically, colours are generated by birefringent crystals such as quartz with ordered arrangement of atoms, or liquid crystals with ordered alignment of electrically polarized molecules. Birefringent crystals split plane-polarized white light - containing all the frequencies in the visible spectrum from red to violet - into two orthogonally oriented rays, one propagating more slowly than the other. When the two rays are recombined with a second polarizer (analyser), the two rays interfere, and that's how colours are generated. To amplify the effect, a full wave plate - the wavelength of green light - is added, hence the characteristic pink background.

We had stumbled upon a new setting for the wave plate that is especially good for biological liquid crystals with low birefringence. Instead of positioning the vibrating directions of the wave plate at the usual $45^{\circ}$ to the polarizers, we placed it at a small angle of $7.5^{\circ}$, which greatly improved the contrast [1-3].

The rainbow in the worm means that the organism is liquid crystalline and coherent to a high degree, even quantum coherent, as consistent with other evidence (see below). It means that the entire organism is electrically polarized from head to tail, like a single uniaxial crystal. Not only are the macromolecules in all the tissues and cells perfectly aligned, but also the $80 \%$ by weight of water in the organism [4]. It is actually the water that makes the entire organism liquid crystalline because this water is liquid crystalline, as others also discovered later (see below). To see the rainbow colours in the living organism, the liquid crystalline molecules not only have to be fully aligned, but also moving coherently, macromolecules and water molecules together. (This has been demonstrated recently by Nuclear Magnetic Resonance (NMR) measurements for a population of water molecules associated with a single molecule of ubiquitin protein trapped inside a reverse micelle, which move in concert with different parts of the protein at rates varying by more than ten orders of magnitude from one region to another [5]). As coherent molecular motions are much slower than visible light vibrations, the ordered alignment of molecules will still be registered by the light transmitted. The most active parts are always the brightest, indicating that the molecular motions are the most coherent. The action is similar to using a very sensitive camera with ultra-short exposure time to capture sharp images of 


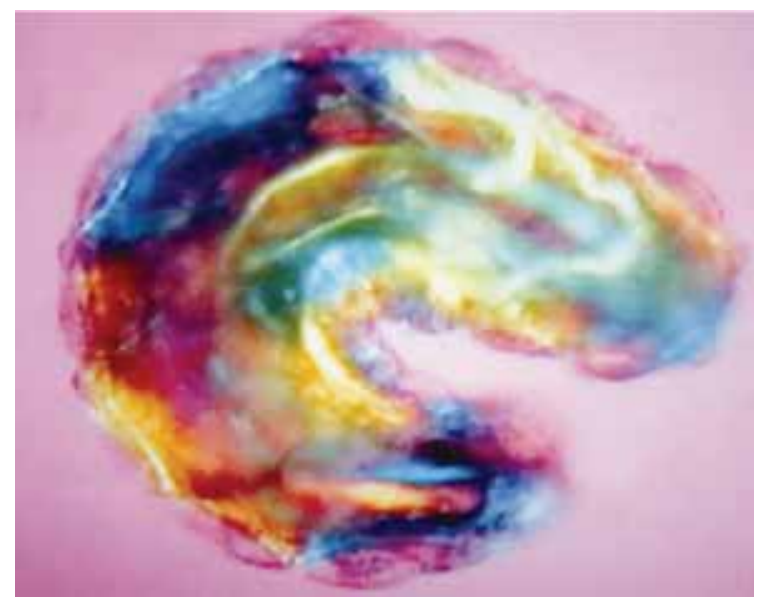

Figure 1: A first instar fruit-fly larva freshly hatched from its egg under the polarizing light microscope.

a fast moving object. In other words, the colour images of live organisms are direct evidence of coherent molecular motions in the organism that depend absolutely on the liquid crystalline water.

\section{WATER THE MEDIUM OF LIFE}

The liquid crystalline living water enables macromolecules to function as quantum molecular machines that transfer and transform energy at close to $100 \%$ efficiency. If not for that, life would be impossible. Water is the supreme medium for life. The archetypal quantum molecular energy machine is the enzyme. Enzymes speed up chemical reactions in organisms by a factor of $10^{10}$ to $10^{23}$ [6], but they cannot do it without water [7], although the role of water is still hardly recognized in the conventional biochemical community. Water gives flexibility to proteins, reduces the energy barrier between reactants and products and increases the probability of quantum tunnelling by a transient compression of the energy barrier.

The rainbow worm was the immediate inspiration for [8] The Rainbow and the Worm, The Physics of Organisms, first published in 1993 and now in its 3rd 2008 edition. It presents empirical evidence and theoretical arguments in support of the idea that the organism is quantum coherent, and that liquid crystalline water plays the lead in creating and maintaining the coherence of organisms. In the Rainbow Worm, I define quantum coherence after Glauber [9] in terms of factorizability, which is later expressed as follows [10, p. 40]: a system is quantum coherent when its parts are so perfectly correlated that their cross-correlations factorize exactly as the product of the individual self-correlations, so that each appears paradoxically as though totally uncorrelated with the rest. It is a state of maximum local freedom and global cohesion; something that's impossible in a classical mechanical system.

Quantum coherence is a sublime state of wholeness; a superposition of coherent activities over all space-times, a pure (ideal) dynamic state towards which the system tends to return asymptotically. I use the idea of 'quantum jazz' to highlight the immense diversity and multiplicity of supramolecular, molecular and submolecular players, the complexity and the coherence of the performance, and above all, the freedom and spontaneity, with each and every player improvising from moment to moment yet keeping in step and in tune with the whole.

Quantum coherent energy storage and mobilization over all space-times also dovetails with a circular thermodynamics of organisms (presented in the same book) that approaches the zeroentropy ideal simultaneously under both equilibrium and far-from equilibrium conditions [11], which is also a good model of sustainable ecological/economic systems. That does not necessarily 
imply that sustainable ecosystems are quantum coherent. Nevertheless, there are quantum physicists who regard the entire universe quantum and quantum coherent [12] as I do [8]; and the reason we find the macroscopic world behaves classically is because we have interacted with it in predominantly classical mechanistic fashion.

But the full extent to which life, the universe and everything depends on water is still unfolding. Astronomers now think water is actually the most abundant substance in the universe, and is present at the birth of the universe [13]. Could it be that the universe too, is powered by water electricity, just as the organism is powered by water electricity, as I shall presently show?

I wrote [10] Living Rainbow $\mathrm{H}_{2} \mathrm{O}$ focussing on water in living organisms as a sequel to the Rainbow Worm [8]. Water scarcity is now threatening the survival of people and planet, especially in times of climate change [14]. Yet, most people still do not know why it is so essential for life, and hence how precious it really is, and why the quality of water is so important. The new book is a synthesis of the recent findings in the quantum physics and chemistry of water that unlock some of the mysteries of why it is so fit for life. It is 'the means, medium and message of life, the beautiful rainbow within that mirrors the one in the sky'.

\section{WATER IS WEIRD AND WONDERFULLY FIT FOR LIFE}

The water molecule is a dipole with separated positive and negative charges associated with the oxygen and the two hydrogen atoms, respectively, so it can engage in dipole interactions with other molecules of water or other dipoles. However, it prefers to hydrogen-bond whenever possible, where the hydrogen atom of one molecule is shared between two oxygen atoms in neighbouring molecules. The favoured configuration is a tetrahedron in which a molecule accepts two hydrogen atoms and donates two hydrogen atoms to neighbouring molecules. It is estimated that at ordinary temperatures and pressures, up to $90 \%$ of the water molecules are hydrogen-bonded [15], although the hydrogen bonds flicker on and off randomly in a matter of pico $\left(10^{-12}\right)$ seconds.

Water is notorious for a host of anomalous properties (some listed in Box) attributed to its propensity to form hydrogen-bonds. The same anomalies are widely regarded as precisely the qualities that make water fit and essential for life.

\section{Major anomalies of water $[16,17]$}

- Neighbours of oxygen form gases with hydrogen at ordinary temperatures and pressures, but water boils at $100^{\circ} \mathrm{C}$ and only freezes at $0^{\circ} \mathrm{C}$ under standard atmospheric pressure, which means organisms are composed of and bathed in liquid water on earth.

- Water has a high heat capacity and high thermal conductivity, thereby preventing temperature fluctuations, enabling organisms to control their body temperature and large bodies of water such as oceans and seas to serve as heat reservoirs, thereby moderating our climate.

- Other liquids increase in density on becoming solid, but ice is lighter than water and floats on it, most fortunately for fish and other aquatic inhabitants.

- Liquid water can be supercooled below $0^{\circ} \mathrm{C}$ without freezing, but on heating, the supercooled liquid does not expand like other liquids; instead it contracts to a maximum density at about $4^{\circ} \mathrm{C}$; this is very important for the hydrological cycle [18] as it plays a key role in rain water percolating underground to refill the aquifers.

- Water's compressibility atypically decreases with increasing temperature reaching a minimum at about $46.5^{\circ} \mathrm{C}$.

- At ordinary temperatures below $35^{\circ} \mathrm{C}$, increasing pressure results in decreased viscosity, which facilitates flow, again at odds with other liquids. 


\section{QUANTUM DELOCALISATION OF THE HYDROGEN BOND}

The key to water's remarkable properties is the hydrogen-bond interconnecting water molecules. It is usually regarded as classical and electrostatic; but many observations are inconsistent with that picture.

Nobel laureate chemist Linus Pauling (1901-1994) first suggested in 1935 that the hydrogen bond and covalent bond in ice may switch places between two neighbours on either side in view of residual entropy (randomness) existing even at very low temperatures [19], and thus, the hydrogen bond must be at least partly covalent.

In 1999, the hydrogen bond in ordinary ice $\mathrm{I}_{\mathrm{h}}$ was probed with inelastic X-ray scattering at the European Synchrotron Radiation Facility of Grenoble [20]. Beams of X-rays are bounced off electrons so both the energy of the electron and the X-ray are changed. The intensity of scattering was measured as a function of energy or momentum at different orientations of a carefully prepared slab of ice and the anisotropy plotted. The results were in good agreement with the predictions based on a fully quantum mechanical model, while predictions based on the classical electrostatic model did not agree with the data at all (Fig. 2).

In the same year, time-resolved pump-probe laser spectroscopy on liquid water at room temperature and pressure revealed resonant intermolecular transfer of $\mathrm{OH}$-stretch excitations mediated by dipole-dipole interactions that was substantially faster than the classical Förster mechanism would predict [21], which again suggested a quantum mechanism.

Further experiments a few years later showed that not only did the electron of the hydrogen bond fail to conform to the classical electrostatic model; the proton too was quantum mechanical. Using ultrafast femto-second $\left(10^{-15} \mathrm{~s}\right)$ pulses of infrared light to excite and probe the O-H covalent bond vibration in liquid water [22], researchers found that only quantum mechanical calculation of the vibrational wave functions could reproduce the experimental absorption spectrum. The excited proton was simultaneously (delocalized) at the $\mathrm{O}-\mathrm{H}$ bond distance from either of two neighbouring oxygen atoms (belonging to two different water molecules) (Fig. 3).

The excited $\mathrm{v}=2$ state for proton delocalization is $6500 \mathrm{~cm}^{-1}(0.82 \mathrm{eV})$, less than $20 \%$ the $\mathrm{O}-\mathrm{H}$ bond energy of $38,750 \mathrm{~cm}^{-1}(4.8 \mathrm{eV})$. The delocalization of protons in water increases the probability of proton transfer, which may play a crucial role in intercommunication (see later).

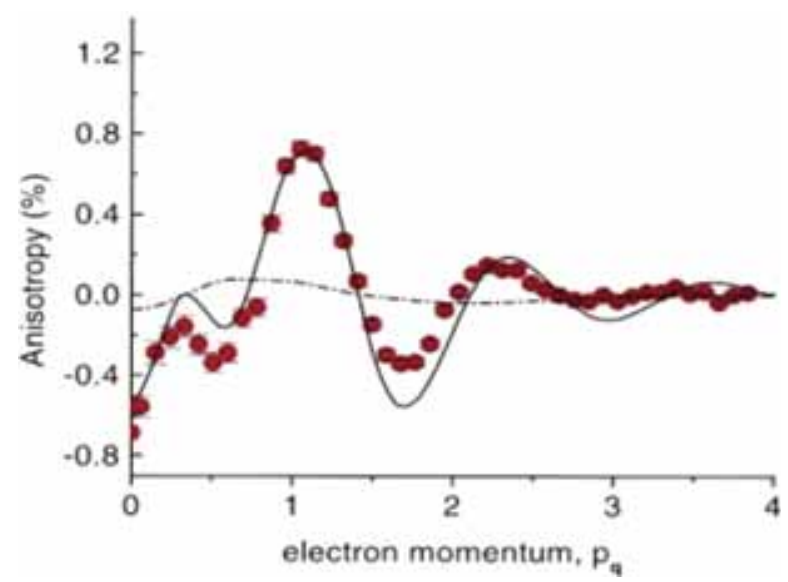

Figure 2: Anisotropy versus electron momentum; solid line quantum model, dotted line classical model, filled circles data (simplified from [20]). 


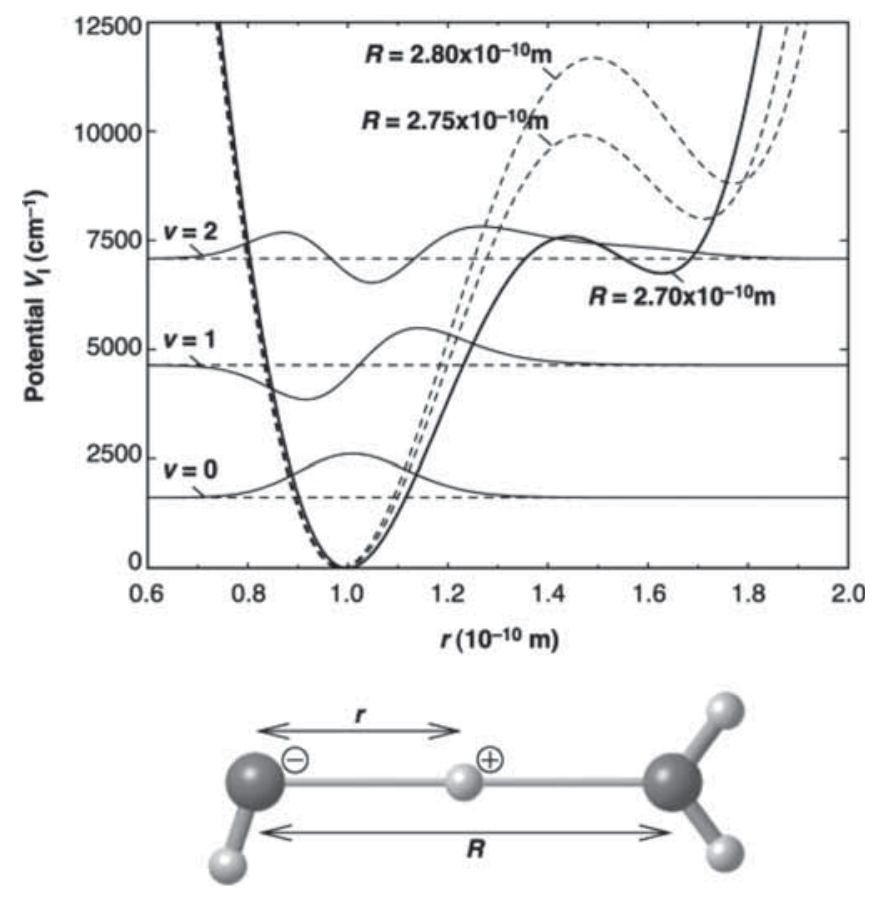

Figure 3: Excitation of the $\mathrm{O}-\mathrm{H}$ bond and delocalization of the proton [22]; the three lowest energy $(\mathrm{v}=0,1,2)$ vibrational wave functions in the potential for $\mathrm{R}(\mathrm{O}-\mathrm{O}$ distance $)=2.7 \AA$.

\section{QUANTUM COHERENT WATER MAKES LIFE ON EARTH}

Standard quantum theory does not predict quantum coherence for liquid water, largely because it ignores both quantum fluctuations and the interaction between matter and electromagnetic field; these are only taken into account in quantum electrodynamics field theory. But conventional quantum electrodynamics field theory applies only to gases.

Giuliano Preparata (1942-2000), Emilio Del Giudice and others at University of Milan Italy, extended conventional quantum electrodynamics theory to the condensed phase of liquids; they showed that interaction between the vacuum (or ambient) electromagnetic field and liquid water induces the formation of large, stable coherent domains (CDs) of about $100 \mathrm{~nm}$ in diameter at ordinary temperature and pressure, and these CDs may be responsible for all the special properties of water including life itself [23-27]. In particular, they showed that the propensity to form tetrahedral directed hydrogen bonds is a consequence of the excited state of water in the CDs that would not happen otherwise.

The $\mathrm{CD}$ is a resonating cavity produced by the electromagnetic field that ends up trapping the field because the photon acquires an imaginary mass, so the frequency of the CD electromagnetic field becomes much smaller than the frequency of the free field with the same wavelength. In other words, as water is a much denser medium than the vacuum or air, electromagnetic fields of the same wavelength will vibrate at a much lower frequency.

Under ambient conditions, water is an approximately equal mixture of CDs surrounded by incoherent regions. This picture, according to Del Giudice and colleagues, is reflected in the many observations supporting a two-state model of liquid water (see [16, 28, 29]). (It is perhaps more 
accurate to say that the water molecules are dancing between the tetrahedral configuration corresponding to the $\mathrm{CDs}$ and non-tetrahedral configuration, so both the $\mathrm{CD}$ and non-CD molecules are interchangeable.)

What is special about water is that the coherent oscillation occurs between the ground state and an excited state at $12.06 \mathrm{eV}$, just below the ionizing threshold of water at $12.60 \mathrm{eV}$. In liquid water, the $\mathrm{CD}$ of about $100 \mathrm{~nm}$ in diameter contains millions of water molecules, and hence close to a million almost free electrons (the fraction of molecules in the excited state is estimated to be 0.13 [26]) that can be donated readily to electron acceptors. It is this property that crucially makes water the means of life and the message of life.

\section{WATER THE MEANS OF LIFE}

Water is the basis of the energy metabolism that powers all living processes; it is the chemistry, the means of life. Water is also the electricity of life for intercommunication, it is both the message and messenger (see next section).

The abundant life on earth, including the human species, depends on photosynthesis in green plants, algae and cyanobacteria that traps the energy of sunlight by means of chlorophyll to split water into hydrogen, electrons and oxygen eqn (1), giving life access to an enormous, practically unlimited energy source, and more importantly, liberating oxygen for the evolution of teaming millions of air-breathing species that filled the earth. (If water is the most abundant substance in the universe, could there be abundant extra-terrestrial beings not too different from earthlings in the rest of the universe?)

$$
\mathrm{H}_{2} \mathrm{O} \rightarrow 2 \mathrm{H}^{+}+2 \mathrm{e}^{-}+\mathrm{O}
$$

The hydrogen ion (protons) and electrons go to reduce (fix) carbon dioxide into carbohydrates and the biomass of photosynthetic organisms, which serve as food for herbivores, and down the food web to include the vast majority of air-breathers. During respiration, carbohydrates are broken down by oxidation (with oxygen) in mitochondria to release energy for growth and reproduction, regenerating carbon dioxide and water [10]. This completes the living dynamo of photosynthesis and respiration, the magic roundabout that turns inanimate substances into living organisms (Fig. 4).

However, it takes $12.6 \mathrm{eV}$ to split water, an energetic photon in the soft $\mathrm{X}$-ray region that would destroy life, and is not what green plants and cyanobacteria use. They use mainly red and to some extent blue light in the visible spectrum.

More than 50 years ago, Nobel Laureate biochemist Albert Szent-Györgyi already suggested [30] that water at interfaces was the key to life. He proposed that water at interfaces such as membranes is in the excited state, which requires considerably less energy to split than water in the ground state. Most if not all water in living organisms is interfacial water, as it is almost never further away from surfaces such as membranes or macromolecules than a fraction of a micron. This is the 'liquid crystalline water' mentioned at the beginning of this article, which is present in all organisms, animals and plants. And chloroplasts where water is split are particularly rich in membrane surfaces, as are mitochondria, where water is regenerated.

A sign that interfacial water is excited is a voltage difference at the boundary between interfacial water and bulk water, as predicted by Szent-Györgyi [30]; and that was observed not long after his proposal (see [24]).

A vivid demonstration of interfacial water hundreds of microns thick next to the surface of a hydrophilic gel was presented by Gerald Pollack and his team at University of Washington Seattle [31] (see also [32-34]), which excludes microspheres as well as other solutes such as proteins and dyes, and hence referred to as an 'exclusion zone' (EZ). 


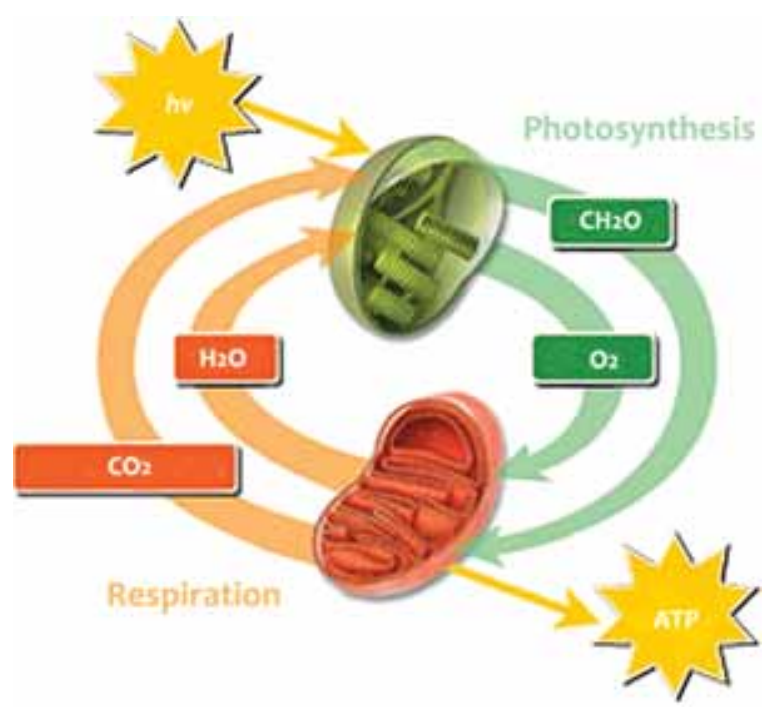

Figure 4: The dynamo of life running on water.

Del Giudice and colleagues [25] suggest that EZ water is in fact a giant CD stabilized on the surface of the hydrophilic gel. As coherent water is excited water with a large collective of almost free electrons, it can easily transfer electrons to molecules on its surface. The boundary between fully coherent interfacial water and normal bulk water becomes a 'redox pile'. In line with this proposal, $\mathrm{EZ}$ water does have a potential difference of $-100 \mathrm{mV}$ to $-200 \mathrm{mV}$ with reference to the bulk water, and can act as a battery $[34,35]$. Pollack's explanation for the formation of EZ is based purely on classical physics, and converges with Del Giudice's quantum field theoretical explanation. This convergence between classical and quantum description is itself a sign of quantum coherence

\section{WATER THE MESSAGE AND MESSENGER OF LIFE - SUPERCONDUCTING PROTON CURRENTS}

The core chemistry of life is reduction-oxidation or redox reactions that transfer electrons between chemical species. The movement of electrons is nothing if not an electric current. However, water electricity is special in that it also involves the movement of positive charges, i.e. protons [36, 37]. Water conducts protons by 'jumping' down a chain of water molecules connected by hydrogen bonds (Fig. 5). A proton leaps on one end of the chain, and a second leaps off at the other end, while electrons are displaced in the other direction. This is much faster than an ordinary electric current involving the flow of electrons.

Structured water confined in carbon nanotubes less than $5 \mathrm{~nm}$ in diameter was demonstrated using high resolution transmission microscopy accompanied by parallel modelling with a Hyperchem software package. The water appeared completely different from that confined in larger nanotubes $[38,39]$ (Fig. 6). I suggested that water confined in the small diameter nanotube, being more ordered, could be superconducting because proton jump-conduction could occur simultaneously down multiple chains of hydrogen-bonded water molecules [40].

Later, Fullerton and colleagues offered a convincing model of liquid crystalline 6-member diameter nanotubes of water interwoven with the triple-helix molecules of collagen molecules in the 
<smiles>OCCOCCOCCO</smiles><smiles>[CH2+]OC=COCOC=COCO</smiles><smiles>OCCOCCO</smiles>

Figure 5: Proton jump conduction down a string of hydrogen bonded water molecules [8].

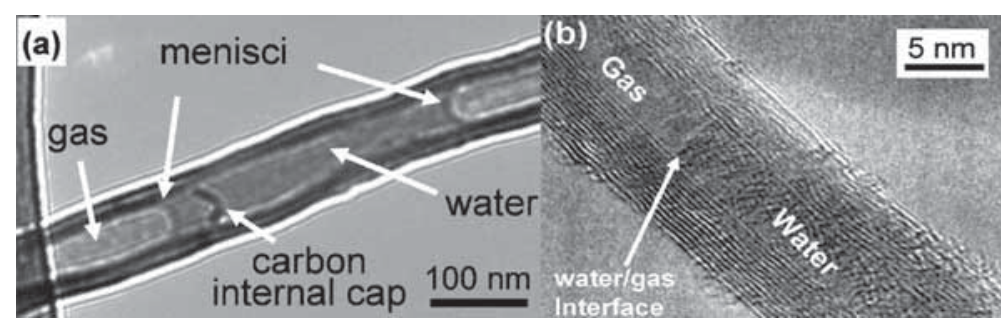

Figure 6: Structured water confined in narrow nanotube (right) compared with ordinary water in wide nanotube (left) (rearranged from [38]).

collagen fibres [41] (Fig. 7) reminiscent of those identified in the small diameter carbon nanotubes; suggesting that these water structures in the extracellular matrix could also be superconducting [42].

It has been known since the 1970s that collagen supports jump conduction of protons and proton conductivity goes up exponentially with water content. Bardelmeyer [43] found that electrical conductivity in bovine Achilles tendon is fully determined by hydration, and the current is primarily carried by protons at water contents up to $45 \%$, and by small ions at water contents beyond $65 \%$. Between water contents of $8.5 \%$ and $126 \%$, conductivity went up eight orders of magnitude. He also estimated that pure water's dissociation constant is $10^{-5}$ that of absorbed water; i.e. adsorbed water is more likely to let go of protons. Similarly, Sasaki found that the conductivity of collagen increased markedly with water absorbed - at an exponent of 5.1-5.4 - between a water content of $0.1-0.3 \mathrm{~g} / \mathrm{g}$ [44], suggesting that continuous chains (of five or more ordered water molecules) adsorbed in collagen enable proton jump conduction to take place.

As seen earlier, the proton is in a delocalized quantum state even in bulk water under ambient conditions. This delocalization has been confirmed recently for water confined in range of hydrophilic gels (reviewed in [45]). Delocalized protons mean jump conduction can be very fast indeed.

Nafion is a synthetic polymer used as a proton exchange membrane. Its chemical structure is a hydrophobic backbone with hydrophilic side chains. When hydrated, it forms channels of inverse 


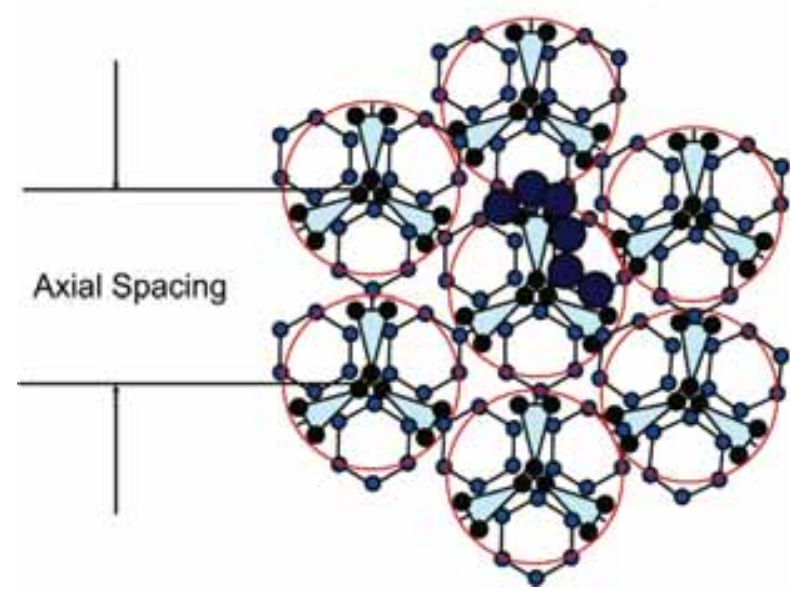

Figure 7: Collagen water structure revealed, cross-section of a fibril consisting of seven triple helical collagen molecules with 6-member water nanotubes fitting within the grooves of the triple helices (simplified from [41]).

micelles with hydrophilic groups facing the cavity and hydrophobic groups facing out. This structure is a good model of the living cell. The interstices between fibres of the cytoskeleton and cytoplasmic membranes form inverse micelle nanospaces and channels that drastically alter enzyme/substrate relationships, often greatly enhancing enzyme activity compared with bulk phase thermodynamic models that still dominate conventional cell biology (see Chapter 18 of Living Rainbow $\mathrm{H}_{2} \mathrm{O}$ [10]).

When Nafion was drawn out into fibres by electro-spinning, the proton conductivity of fibres with diameters $>2 \mu \mathrm{m}$ was similar to the bulk Nafion film $(\sim 0.1 \mathrm{~S} / \mathrm{cm})$. However, when the fibre diameter was $<1 \mu \mathrm{m}$, proton conductivity rose sharply with decreasing fibre diameter and reached $1.5 \mathrm{~S} / \mathrm{cm}$ for the $400 \mathrm{~nm}$ diameter fibre, an order of magnitude higher than the bulk Nafion film, or silicon, a semi-conductor. Conductivity of the fibre also increased a 100 -fold as relative humidity rose from $50 \%$ to $90 \%$; in comparison, conductivity of the bulk film increased only 10-fold [46].

The inverse micelle model may be even more relevant to the extracellular milieu of multicellular animals, which is traversed by collagen fibres consisting of fibrils interwoven with nanotubes of water (Fig. 7). These water channels aligned with collagen fibres are most likely the anatomical correlates of the acupuncture meridians of traditional Chinese medicine, as I and David Knight first suggested in 1998 [47]; the hypothesis is still much alive and untested [48].

What one needs to imagine are proton and electron currents flowing inside the cells and over extracellular distances, delivering physical and chemical messages concerning the redox status, setting in motion the requisite core chemical reactions that restore local and global energy balance (and also the peripheral chemistry that forms the basis of the highly nuanced passions and feelings that make life so exciting for organisms).

\section{THE ELETROMAGENTIC LANGUAGE OF CELLS AND MOLECULES}

Finally, how do cells and molecules actually find one another? Conventional wisdom says hormones and receptors, cell-cell recognition molecules, and lock-and-key principle for molecules that somehow bump into each other at random (see current authoritative textbook Molecular Biology of the Cell [49]). 
Actually, there is substantial evidence that molecules find each other by electromagnetic fields, by resonating to common frequencies $[8,10]$. Molecules that react together were found to share a common frequency; which is thought to be how they can attract each other (reviewed in [50]). This makes even more sense in the context of quantum coherent water.

Del Giudice and colleagues [24-26] argue that water CDs can be easily excited, and are able to capture surrounding electromagnetic fields to produce coherent excitation in the frequencies of the external fields. This in turn enables selective coherent energy transfer to take place. All molecules have their own spectrum of vibrational frequencies. If the molecule's spectrum contain a frequency matching that of the water $\mathrm{CD}$, it would get attracted to the $\mathrm{CD}$, and become a guest participant in the CDs coherent oscillation, and settle on the CDs surface. Furthermore, the CDs excitation energy would become available to the guest molecules as activation energy for the chemical reactions.

Is it possible that cells or organisms as a whole also intercommunicate by means of electromagnetic and electric signals? This is completely uncharted territory as far as conventional cell biology is concerned. It is the water in us that gives us life and makes us sensitive to electromagnetic fields; there is a distinct possibility that we are sensitive to the fields of other organisms, as we are sensitive to fields of the sun and the earth (reviewed in [51]), and possibly also from distant stars; all without our conscious knowledge.

To conclude, life appears quantum electro-dynamical through and through, and water is at the heart of it all. A whole new vista has opened up thanks to all the dedicated water scientists who have contributed to this vision and whose work I have cited; and especially Albert Szent-Györgyi [29] and Gilbert Ling [52], both of whom recognized the central role of water in producing and animating life more than half a century ago. Our adventures have only just begun.

\section{REFERENCES}

[1] Ho, M.W. \& Lawrence, M., Interference colour vital imaging: a novel noninvasive technology. Microscope and Analysis, September, p. 26, 1993.

[2] Ho, M.W. \& Saunders, P.T., Liquid crystalline mesophase in living organisms. Bioelectrodynamics and Biocommunication, eds. M.W. Ho, F.A. Popp \& U. Warnke, World Scientific: Singapore, pp. 213-228, 1994. doi: http://dx.doi.org/10.1142/97898145038220008

[3] Ross, S., Newton, R.H., Zhou, Y.M., Haffegee, J., Ho, M.W., Bolton, J. \& Knight, D., Quantitative image analysis of birefringent biological materials. Journal of Microscopy, 187, pp. 62-67, 1997. doi: http://dx.doi.org/10.1046/j.1365-2818.1997.2160776.x

[4] Ho, M.W., Zhou, Y.M., Haffegee, J., Watton, A., Musumeci, F., Privitera, G., Scordino, A. \& Triglia, A., The liquid crystalline organism and biological water. Water in Cell Biology, eds. G. Pollack, I.L. Cameron \& D.N. Wheatley, Springer: Dordrecht, pp. 219-251, 2006.

[5] Nucci, N.V., Pometun, M.S. \& Wand, A.J., Site-resolved measurement of water-protein interaction by solution NMR. Natural Structural Molecular Biology, 18, pp. 245-250, 2010. doi: http://dx.doi.org/10.1038/nsmb.1955

[6] Kraut, D.A., Carroll, K.S. \& Herschlag, D., Challenges in enzyme mechanism and energetics. Annual Review Biochemistry, 72, pp. 517-571, 2003. doi: http://dx.doi.org/10.1146/annurev. biochem.72.121801.161617

[7] Ho, M.W., Dancing with macromolecules. Science in Society, 48, pp. 11-15, 2010. doi: http:// dx.doi.org/10.1142/6928

[8] Ho, M.W., The Rainbow and the Worm, the Physics of Organisms, 3rd edn., World Scientific: Singapore, 2008.

[9] Glauber, R.J., The quantum theory of optical coherence. Physical Review, 130, pp. 2529-2539, 1963. doi: http://dx.doi.org/10.1103/PhysRev.130.2529 
[10] Ho, M.W., Living Rainbow $\mathrm{H}_{2} \mathrm{O}$, World Scientific and Imperial College Press: Singapore, 2012.

[11] Ho, M.W., Circular economy of organisms and sustainable systems. Systems, 1, pp. 30-49, 2013. doi: http://dx.doi.org/10.3390/systems 1030030

[12] Albrecht, A., Some remarks on quantum coherence. Journal of Modern Optics, 41, p. 2457, 1994. doi: http://dx.doi.org/10.1080/09500349414552311

[13] Astronomers find largest, most distant reservoir of water, Mission News, NASA, available at http://www.nasa.gov/topics/universe/features/universe20110722.html, 22 July 2011.

[14] Ho, M.W., World water supply in jeopardy. Science in Society, 56, pp. 38-43, 2012.

[15] Bakker, H.J. \& Skinner J.L., Vibrational spectroscopy as a probe of structure and dynamics of water. Chemical Reviews, 110, pp. 1498-1517, 2010. doi: http://dx.doi.org/10.1021/cr9001879

[16] Chaplin, M., What is liquid water. Science in Society, 58, pp. 41-45, 2013.

[17] Vedamuthu, M., Singh, S. \& Robinson, C.W., Properties of liquid water: origin of the density anomalies. Journal of Physical Chemistry, 98, pp. 2222-2230, 1994. doi: http://dx.doi. org/10.1021/j100060a002

[18] Coats, C., Living Energies, Gateway Books: Bath, UK, 1996.

[19] Pauling, L., The structure and entropy of ice and of other crystals with some randomness of atomic arrangement. Journal of American Chemical Society, 57, pp. 2680-2684, 1935. doi: http://dx.doi.org/10.1021/ja01315a102

[20] Isaacs, E.D., Shukla, A., Platzman, P.M., Hamann, D.R., Barbiellin, B. \& Tulk, C.A., Covalency of the hydrogen bond in ice: a direct X-ray measurement. Physical Review Letters, 82, pp. 600-603, 1999. doi: http://dx.doi.org/10.1103/PhysRevLett.82.600

[21] Woutersen, S. \& Bakker, H.J., Resonant intermolecular transfer of vibrational energy in liquid water. Nature, 402, pp. 507-509, 1999. doi: http://dx.doi.org/10.1038/990058

[22] Bakker, H.J. \& Nienhuys, H.K., Delocalization of protons in liquid water. Science, 297, pp. 587-590, 2002. doi: http://dx.doi.org/10.1126/science.1073298

[23] Arani, R., Bono, I., Del Giudice, E. \& Preparata, G., QED coherence and the thermodynamics of the water. International Journal of Modern Physics B, 9, pp. 1813-1841, 1995. doi: http:// dx.doi.org/10.1142/S0217979295000744

[24] Del Giudice, E., Old and new views on the structure of matter and the special case of living matter. Journal of Physics: Conference Series, 67, p. 012006, 2007.

[25] Del Giudice, E., Spinetti, P.R. \& Tedeschi, A., Water dynamics at the root of metamorphosis in living organisms. Water, 2, pp. 566-586, 2010. doi: http://dx.doi.org/10.3390/w2030566

[26] Del Giudice, E. \& Pulselli, R.M., Formation of dissipative structures in liquid water. International Journal of Design \& Nature and Ecodynamics, 5, pp. 21-26, 2010. doi: http://dx.doi. org/10.2495/DNE-V5-N1-21-26

[27] Ho, M.W., Quantum coherent water \& life. Science in Society, 51, pp. 26-28, 2011.

[28] Ho, M.W., Two-states water explains all? Science in Society, 32, pp. 17-18, 2006.

[29] Huang, C., Wikfeldt, K.T., Tokushima, T. \& Nilsson, A., The inhomogeneous structure of water at ambient conditions. Proc Natl Acad Sci USA, 106, pp. 15214-15218, 2009. doi: http:// dx.doi.org/10.1073/pnas.0904743106

[30] Szent-Györgyi, A., Introduction to a Supramolecular Biology, Academic Press: New York, 1960.

[31] Zheng, J.M. \& Pollack, G.H., Long-range forces extending from the polymer gel surfaces. Physical Review E, 68, pp. 314-318, 2003. doi: http://dx.doi.org/10.1103/PhysRevE.68.031408

[32] Ho, M.W., Water forms massive exclusion zones. Science in Society, 23, pp. 50-51, 2004.

[33] Pollack, G.H., Water, energy and life: fresh views from the water's edge. International Journal of Design \& Nature and Ecodynamics, 5, pp. 27-29, 2010. doi: http://dx.doi.org/10.2495/ DNE-V5-N1-27-29 
[34] Pollack, G.H., The Fourth Phase of Water, Ebner \& Sons Publishers: Seattle, Washington, 2013.

[35] Ho, M.W., Liquid crystalline water at the interface. Science in Society, 38, pp. 37-39, 2008.

[36] Riistama, S., Hummer, G., Puustinen, A., Dyer, R.B., Woodruff, W.H. \& Sikatrom, M., Bound water in the proton translocation mechanism of the haem-copper oxidation. FEBS Letters, $\mathbf{4 1 4}$, pp. 275-289, 1997. doi: http://dx.doi.org/10.1016/S0014-5793(97)01003-X

[37] Ho, M.W., Positive electricity zaps through water chains. Science in Society, 28, pp. 49-50, 2005.

[38] Ye, H., Naguib, N. \& Gogotsi, Y., TEM study of water in carbon nanotubes. JEOL News, 39, pp. 2-7, 2004.

[39] Naguib, N., Ye, H., Gogtsi, Y., Yazicioglu, A.G., Megaridis, C.M. \& Yoshimura, M., Observation of water confined in nanometer channels of closed carbon nanotubes. Nano Letters, $\mathbf{4}$, pp. 2237-2243, 2004. doi: http://dx.doi.org/10.1021/n10484907

[40] Ho, M.W., First sighting of structured water. Science in Society, 28, pp. 47-48, 2005.

[41] Fullerton, G.D. \& Amurao, M.R., Evidence that collagen and tendon have monolayer water coverage in the native state. International Journal of Cell Biology, 30, pp. 56-65, 2006. doi: http://dx.doi.org/10.1016/j.cellbi.2005.09.008

[42] Ho, M.W., Collagen water structure revealed. Science in Society, 32, pp. 15-16, 2006.

[43] Bardelmeyer, G.H., Electrical conduction in hydrated collagen. I. Conductivity mechanisms. Biopolymers, 12, pp. 2289-2302, 1973. doi: http://dx.doi.org/10.1002/bip.1973.360121008

[44] Sasaki, N., Dielectric properties of slightly hydrated collagen: time-water content superposition analysis. Biopolymers, 23, pp. 1725-1734, 1984. doi: http://dx.doi.org/10.1002/bip.360230908

[45] Ho, M.W., Superconducting quantum coherent water in nanospace confirmed. Science in Society, 55, pp. 48-51, 2012.

[46] Dong, B., Gwee, L., Salas-de la Cruz, D., Winey, K.I. \& Elabd, Y.A., Super proton conductive high-purity Nation nanofibers. Nano Letters, 10, pp. 3785-3790, 2010. doi: http://dx.doi. org $/ 10.1021 / \mathrm{nl} 102581 \mathrm{w}$

[47] Ho, M.W. \& Knight, D.P., The acupuncture system and the liquid crystalline collagen fibers of the connective tissues. American Journal of Chinese Medicine, 26, pp. 251-263, 1998. doi: http://dx.doi.org/10.1142/S0192415X98000294

[48] Ho, M.W., Super-conducting liquid crystalline water aligned with collagen fibres in the fascia as acupuncture meridians of traditional Chinese medicine. Forum on Immunopathological Diseases and Therapeutics, 3, pp. 221-236, 2012. doi: http://dx.doi.org/10.1615/ForumImmunDisTher.2013007869

[49] Lewis, J. \& Raff, M., Molecular Biology of the Cell, Garland Science: New York, 2008.

[50] Ho, M.W., The real bioinformatics revolution. Science in Society, 33, pp. 42-45, 2007.

[51] Ho, M.W., Life is water electric. Science in Society, 57, pp. 43-47, 2013.

[52] Ling, G., Life at the Cell and below Cell Level, Pacific Press: California, USA, 2001. 